\title{
Anomalous Hall effect in antiferromagnetic/nonmagnetic interfaces
}

\author{
M. Asa, ${ }^{1}$ C. Autieri, ${ }^{2,3}$ R. Pazzocco,${ }^{1}$ C. Rinaldi $\odot,{ }^{1}$ W. Brzezicki, ${ }^{3}$ A. Stroppa,${ }^{4}$ M. Cuoco,${ }^{5}$ \\ G. Varvaro, ${ }^{6}$ S. Picozzi, ${ }^{2}$ and M. Cantoni ${ }^{1}{ }^{1, *}$ \\ ${ }^{1}$ Department of Physics, Politecnico di Milano, clo Via G. Colombo 81, 20133, Milano, Italy \\ ${ }^{2}$ Consiglio Nazionale delle Ricerche CNR-SPIN, clo Universitá “G. D’Annunzio”, 66100 Chieti, Italy \\ ${ }^{3}$ International Research Centre MagTop, Institute of Physics, Polish Academy of Sciences, Aleja Lotników 32/46, PL-02668 Warsaw, Poland \\ ${ }^{4}$ Consiglio Nazionale delle Ricerche CNR-SPIN, clo Università L'Aquila, 67100 L'Aquila, Italy \\ ${ }^{5}$ Consiglio Nazionale delle Ricerche CNR-SPIN, clo Universitá di Salerno, Via Giovanni Paolo II, 132 - 84084 - Fisciano (SA), Italy \\ ${ }^{6}$ Consiglio Nazionale delle Ricerche CNR-ISM, Via Salaria km 29.300, Monterotondo Scalo (Roma), 00015, Italy
}

(Received 6 April 2019; revised 30 September 2020; accepted 1 December 2020; published 21 December 2020)

\begin{abstract}
We report a combined theoretical and experimental investigation of magnetic proximity and Hall transport in $\mathrm{Pt} / \mathrm{Cr}$ bilayers. Density-functional theory indicates that interfacial magnetic moments can be induced in the Pt layer and a strong magnetocrystalline anisotropy with an out-of-plane easy axis arises in the antiferromagnet. A signal ascribed to the anomalous Hall effect is detected and associated to the interface between $\mathrm{Pt}$ and $\mathrm{Cr}$ layers. We discuss how this effect originates from the Berry curvature of the electronic structure at the interface and the correlation with the character of the proximity-induced magnetic moments.
\end{abstract}

DOI: 10.1103/PhysRevResearch.2.043394

\section{INTRODUCTION}

The anomalous Hall effect (AHE) is typically associated with ferromagnetic metals because of broken time-reversal symmetry and spin-orbit coupling (SOC) [1]. Recent observations of large AHE originating from a nonvanishing Berry phase in antiferromagnets with noncollinear spin arrangement $[2,3]$ have attracted great interest for possible applications in the emerging field of antiferromagnet spintronics [4]. It is, however, generally accepted that ordinary antiferromagnets with collinear spin configuration should not exhibit AHE due to the vanishing Berry phase except when specific symmetry conditions of the crystals are realized [5].

In this work we prove that interfacing a collinear antiferromagnet (AFM) with a nonmagnetic (NM) metallic layer yields a detectable AHE whose sign is controllable by field cooling (FC) in the NM/AFM system Pt/Cr. We propose that the AHE is substantially driven by the Berry curvature of electronic states at the NM/AFM interface, and the response is not significantly affected by the character of the magnetic proximity effect (MPE) in Pt [6]. Chromium is a prototypical itinerant antiferromagnet with a spin-density-wave (SDW) magnetic ordering [7]. Platinum is nonmagnetic in its bulk form, on the verge of Stoner ferromagnetism. In thin-film heterostructures, MPE has been demonstrated by $\mathrm{x}$-ray magnetic circular

\footnotetext{
*Author to whom correspondence should be addressed: matteo.cantoni@polimi.it

Published by the American Physical Society under the terms of the Creative Commons Attribution 4.0 International license. Further distribution of this work must maintain attribution to the author(s) and the published article's title, journal citation, and DOI.
}

dichroism (XMCD) when Pt is interfaced with ferromagnetic $3 d$ metals [8-11]. MPE has also been invoked to explain some transport properties of $\mathrm{Pt}$ on insulating yttrium iron garnet [12], although this is still debated [13]. More recently, MPE in Pt has been exploited to detect the state of a neighboring magnetoelectric antiferromagnet $\mathrm{Cr}_{2} \mathrm{O}_{3}$ by means of the AHE $[14,15]$, demonstrating the use of $\mathrm{Pt}$ as a valuable probe in the context of the AFM-based spintronics [4].

By combining ab initio calculations and electrical measurements, we demonstrate that a spin-polarized interface forms between Cr and Pt. Density functional theory (DFT) predicts a magnetic moment induced in Pt by neighboring $\mathrm{Cr}$ with out-of-plane (OOP) anisotropy. We show the electrical detection of AHE also in relatively thick metallic heterostructures, despite the interfacial origin of such effects. Exploiting the peculiar transport properties of chromium related to SDW antiferromagnetism $[16,17]$, we can unambiguously associate the presence of AHE to the AFM state of Cr. Finally, we discuss how the AHE and its temperature dependence can be accounted for by the Berry curvature of the interfacial electronic states, which we expect to occur also in other NM/AFM systems. This finding is consistent with the observation of the absence of ferrimagnetism in the $\mathrm{Cr} / \mathrm{Pt}$ system.

\section{METHODS}

To model the $\mathrm{Pt} / \mathrm{Cr}$ interface, we performed first-principles density-functional calculations within the gradient generalized approximation by using the plane-wave VASP [18] package and the Perdew-Burke-Ernzerhof (PBE) for the exchange-correlation functional [19] including SOC. The core and the valence electrons were treated with the projector augmented-wave method [20] and a cutoff of $450 \mathrm{eV}$ for the plane-wave basis. In supercell calculations we use eight 
Cr layers and the lattice constant of Cr bulk (2.89 ̊) (see Appendix A).

Epitaxial $\mathrm{Pt} / \mathrm{Cr}$ bilayers with a sharp and ordered interface were grown by molecular beam epitaxy on $\mathrm{MgO}$ (001) substrates to experimentally verify the presence of AHE. Cr thin films were deposited under ultrahigh-vacuum conditions with thickness ranging from 25 to $75 \mathrm{~nm}$. Details on the growth are reported in Appendix B and Ref. [21]. A Pt overlayer with thickness between 0.6 (four monolayers) and $3 \mathrm{~nm}$ was deposited on top. Optical lithography and ion milling were used on the samples to define 50-micron-wide Greek crosses. The compensation of geometrical offsets in this geometry was achieved by subtracting measurements in which current and voltage contacts are exchanged [22]. At the same time, the current reversal method allows for compensation for thermoelectric offsets (see Appendix C) [23]. The measurement protocol goes as follows: by cooling the sample from above its Néel temperature in an OOP magnetic field $B= \pm 0.4 \mathrm{~T}$ (field cooling), we set the magnetic state of $\mathrm{Cr}$. Subsequently, this state is read by exploiting the anomalous Hall resistance versus temperature, raised with a constant rate of $2 \mathrm{~K} / \mathrm{min}$ in a closed-circuit He cryostat.

\section{RESULTS}

We assume that the SDW in Cr [24] will not affect the properties of the $\mathrm{Pt} / \mathrm{Cr}$ interface due to the small deviation from commensurate antiferromagnetism. In this $3 d-5 d$ interface we neglect the possibility of complex magnetism [25,26], since it was shown that the Dzyaloshinskii-Moriya interaction is negligible at the $\mathrm{Cr} / \mathrm{Pt}$ interface [26].

Bulk Pt presents a van Hove singularity in the density of states close to the Fermi level. In general the interface hybridization and the magnetic proximity tends to favor a Stoner-type ferromagnetic instability [27], so that Pt may undergo a magnetic phase transition at the interfaces. We calculate the profile magnetization of the $\mathrm{Pt}(\mathrm{N}) / \mathrm{Cr}$ heterostructure in the collinear approximation as function of the number $(\mathrm{N})$ of $\mathrm{Pt}$ layers. The $\mathrm{Pt}$ interface always couples ferromagnetically with $\mathrm{Cr}$, as for $3 d$ ferromagnetic materials [28]. The magnetic proximity demonstrates that the spin polarization, arising into the antiferromagnet, penetrates the Pt layers. Although the spatial profile is nonmonotonous, the total magnetization is nonvanishing and of the order of $0.3 \mu_{B}$ (see Appendix D).

The magnetocrystalline anisotropy (MCA) is calculated from first principles (including SOC) as a function of the lattice constant. As illustrated in Fig. 1, the MCA shows a strong sensitivity to strain effects, highlighted by the nonmonotonic behavior and in agreement with what is previously found for $3 d$ ferromagnets interfaced with $5 d$ materials [29]. For the lattice constant of bulk $\mathrm{Cr}$, a large MCA with an OOP easy axis is obtained. The contribution to the MCA mainly comes from the first layer of $\mathrm{Cr}$, as demonstrated by means of DFT evaluation of the density of states (DOS) (see Appendix A).

On the experimental side, the occurrence of a ferrimagnetic CrPt alloy has been excluded in Ref. [21]. A spurious contribution of ferromagnetic character, likely arising from substrate impurities, has been detected (see Appendix E), but it cannot be related to our results because it is substantially

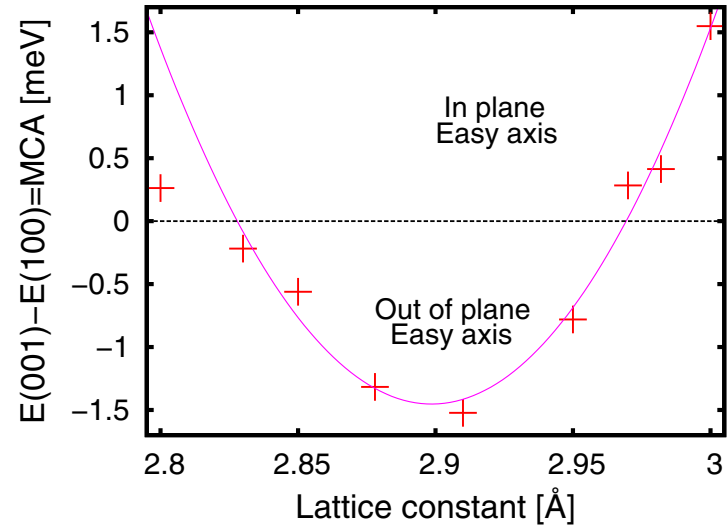

FIG. 1. MCA of the Pt/Cr heterostructure as a function of lattice constant (red crosses). Positive (negative) values of the MCA indicate an in-plane (out-of-plane) easy axis, respectively. The purple line is a guide for the eyes.

temperature independent in the $100-320 \mathrm{~K}$ range. Figure 2 reports the transport properties of a $\operatorname{Pt}(2) / C r(50)$ bilayer (thickness in nanometers) measured with a $\pm 20 \mathrm{~mA}$ probing current. Figure 2(a) shows the longitudinal resistivity $\rho_{x x}$ of the sample (empty dots) and its first derivative with respect to the temperature (black line) in the $20-330 \mathrm{~K}$ temperature (T) range. Here it is crucial to consider that the formation of the SDW in Cr determines the opening of a gap in the Fermi surface along the direction of the wave vector [7]. This results in a reduction of the carrier density, which produces a "kink" in the resistivity versus $T$ curve. Following Ref. [16], the Néel temperature $T_{N}$ can be associated to the local maximum in $d \rho / d T$. From Fig. 2(a) we find $T_{N}=290 \pm 5 \mathrm{~K}$, which is slightly inferior to bulk $\mathrm{Cr} T_{N}=311 \mathrm{~K}$ and comparable to other epitaxial thin films [17].

The same information can be extracted from the ordinary Hall resistivity $\rho_{x y}$, since it is related to the carrier density $n$

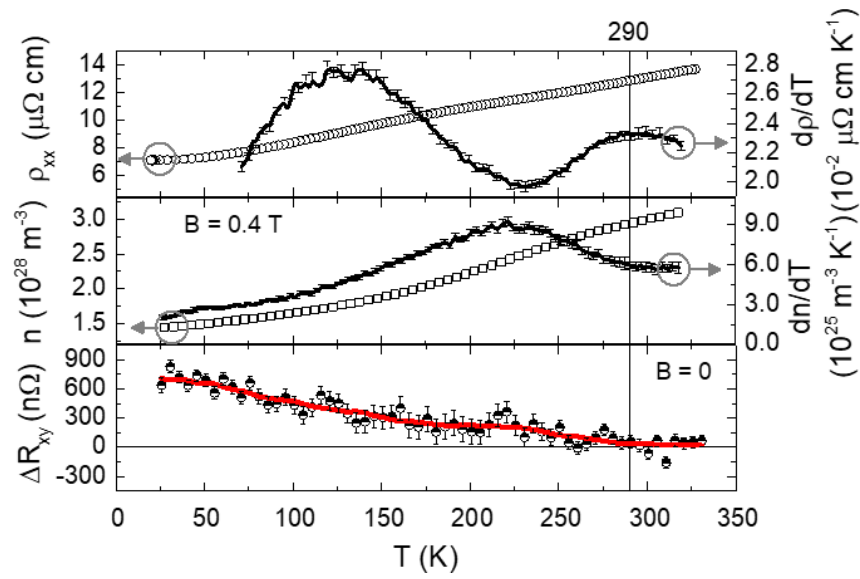

FIG. 2. Transport properties of a $\mathrm{Pt}(2) / \mathrm{Cr}(50)$ Hall cross as a function of temperature: (a) resistivity (empty dots) and first derivative (black line); (b) number of carriers from ordinary Hall measurements (empty squares) and first derivative (black line); (c) anomalous Hall resistance difference taken at two opposite amplitudes of the magnetic field. Error bars indicate the standard error on multiple samples. The red line is a guide for the eye. 
by the simple relation coming from the Drude model $\rho_{x y}=$ $-B / n e$ ( $e$ is the electron charge), where $\rho_{x y}=R_{x y} t$ ( $t$ is the film thickness). The temperature dependence of $n$ (empty squares) and its first derivative (black line) with respect to $T$ are shown in Fig. 2(b). For the reasons given above, the increase of $d n / d T$ below $290 \mathrm{~K}$ is consistent with the AFM phase transition of $\mathrm{Cr}$ [17], in agreement with the value established from longitudinal resistivity measurements.

Finally, in Fig. 2(c) we report the difference $\left(\Delta R_{x y}\right)$ between the anomalous Hall resistances measured at remanence in states prepared by opposite FCs $(B= \pm 0.4 \mathrm{~T})$. Measurements at remanence permits excluding a transverse contribution arising from ordinary Hall effects and isolation of the effect related to the memory of the system achieved through manipulation of the AF state. The small signal, in the order of hundreds of nano-ohms, can be reproducibly detected and is found to depend only on the direction of $B$ during the FC. $\Delta R_{x y}$ decreases with temperature, approaching zero at $T^{*}=290 \mathrm{~K}$, which coincides with the previously determined $T_{N}$, considering the experimental error bar.

Below $T^{*}$ the application of magnetic fields no longer affects the transverse resistance at remanence, thus excluding the possible influence of ferromagnetic contaminations on the signal. On the contrary, the robustness against external fields can be explained by the negligible magnetic susceptibility of $\mathrm{Cr}$ in the AFM phase. This strict correspondence between $\mathrm{Cr}$ magnetic order, established from ordinary transport measurements, and the presence of an AHE clearly relates the origin of the latter to the antiferromagnet state. We emphasize that conventional magnetometry does not allow observation of this phase transition. No change in the magnetic signal is observed across the phase transition on an unpatterned $\operatorname{Pt}(2) / \operatorname{Cr}(50)$ sample with a superconducting quantum interference device (SQUID) (see Appendix E).

A first hint that the effect originates within the Pt layer comes from the comparison of different $\mathrm{Cr}$ and $\mathrm{Pt}$ thicknesses (Fig. 3). $\Delta R_{x y}$ at $20 \mathrm{~K}$ is almost identical for samples with 25-, 50-, and 75-nm-thick $\mathrm{Cr}$ films and 3-nm-thick Pt overlayer, with an average value of $680 \pm 120 \mathrm{n} \Omega$. In the good-metal regime, the anomalous Hall resistance is proportional to the sample magnetization and independent of the resistivity [14]. With $\Delta R_{x y}$ being unrelated to $\mathrm{Cr}$ thickness, a bulk contribution should be excluded. Instead, when a thinner Pt layer $(0.6 \mathrm{~nm}$, corresponding to four monolayers) is considered, a smaller $\Delta R_{x y}=275 \pm 30 n \Omega$ is found between states written with the opposite field. We incidentally note that $\Delta R_{x y}$ of the thinnest $\mathrm{Cr}$ sample $(25 \mathrm{~nm})$ approaches zero already below $200 \mathrm{~K}$. This is consistent with the reported increase of $\mathrm{Cr}$ transition temperature with thickness in epitaxial thin films [16].

A second fact supporting the interfacial origin comes from measurements on the same system with a gold spacer between $\mathrm{Pt}$ and $\mathrm{Cr}$. At variance with $\mathrm{Pt} / \mathrm{Cr}$ systems, proximity-induced moments in $\mathrm{Au} / \mathrm{Cr}$ are not expected in DFT calculations (see Appendix D). This is in excellent agreement with the experimental results reported in Fig. 3: the $\mathrm{Pt}(3) / \mathrm{Au}(3) / \mathrm{Cr}(50)$ sample presents a negligible signal compared to that of $\mathrm{Pt} / \mathrm{Cr}$ samples. Note that the $\mathrm{Pt}(3) / \mathrm{Au}(3) / \mathrm{Cr}(50)$ and $\operatorname{Pt}(3) / \mathrm{Cr}(50)$ heterostructures are from the same deposition (realized with the help of a mechanical shutter). Hence the different sig-

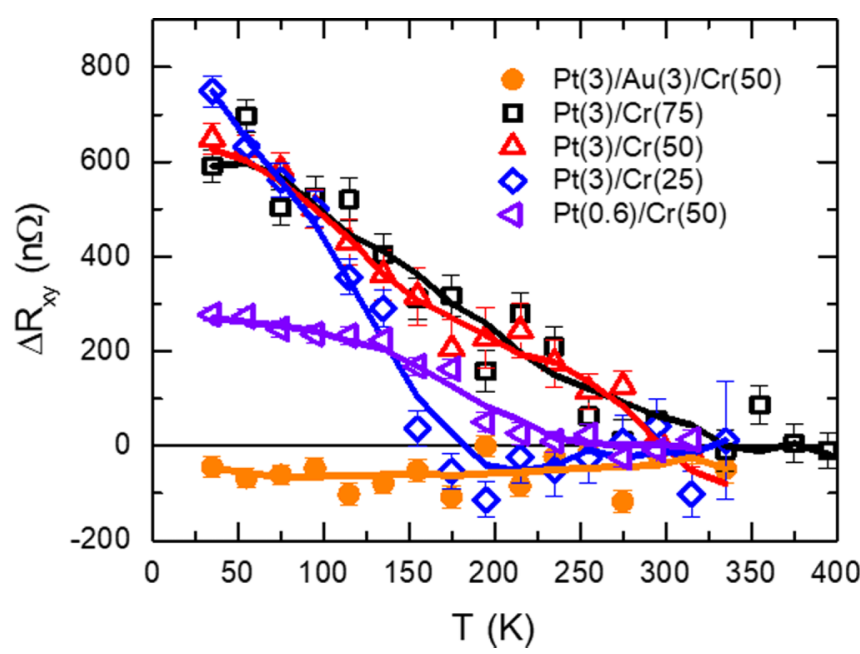

FIG. 3. Difference of the anomalous Hall resistance taken at two opposite values of the magnetic field as a function of temperature in $\mathrm{Pt} / \mathrm{Cr}$ and $\mathrm{Au} / \mathrm{Cr}$ bilayers. Nominal thicknesses are expressed in nanometers. Error bars indicate the standard error on multiple samples. The lines are only a guide for the eye.

nal cannot be ascribed to the quality of $\mathrm{Cr}$, which is indeed the same (see Appendix F). Moreover, the AHE suppression with the interposition of the diamagnetic Au interlayer allows exclusion of any significant contribution to the signal given by spin-Hall magnetoresistance [30], since any phenomena related to spin-current generation in $\mathrm{Pt}$ would be unaffected as the spacer thickness is much thinner than the spin diffusion length in $\mathrm{Au}(\lambda>30 \mathrm{~nm}[31])$.

Whereas the different signal coming from $\mathrm{Pt} / \mathrm{Cr}$ and $\mathrm{Au} / \mathrm{Cr}$ interfaces could be justified with the different proximityinduced magnetization, the temperature trend of the anomalous Hall resistance cannot be uniquely explained as due to this net magnetic moment. In a conventional picture the transverse resistivity in a system with magnetization $M$ follows the relation $[32,33]$

$$
\rho_{x y}=R_{0} H+\alpha M \rho_{x x 0}+\beta M \rho_{x x 0}^{2}+b M \rho_{x x}^{2},
$$

with $\alpha, \beta$, and $b$ being the related to the skew scattering, the side jump, and the intrinsic term, respectively; $R_{0}$ is the ordinary Hall coefficient, $\rho_{x x 0}$ the zero-temperature residual resistivity, and $\rho_{x x}$ the total longitudinal resistivity.

According to the scaling relation for $\rho_{x y}$, the temperature variation of the anomalous Hall resistivity is linked to the thermal dependence of the magnetization and of the longitudinal resistivity. It is worth pointing out that the amplitude of $\rho_{x x}$ is only doubled when moving from 50 to about $300 \mathrm{~K}$ [see Fig. 2(a)]. Such observation implies that the magnetization should play a major role in setting the thermal dependence of the anomalous Hall resistivity. However, a closer inspection of the thermal profile of $\rho_{x y}$ indicates few distinct marks (see Fig. 3). $\rho_{x y}$ does not follow a Curie-Weiss model $\rho_{x y} \propto$ $1 /\left(T-T_{N}\right)$ but, on the contrary, exhibits a smooth switching close to the antiferromagnetic transition. Moreover, the anomalous Hall resistance keeps increasing from 150 to $50 \mathrm{~K}$ in a region where the longitudinal resistivity has only minor variations, and the magnetization due to the magnetic 


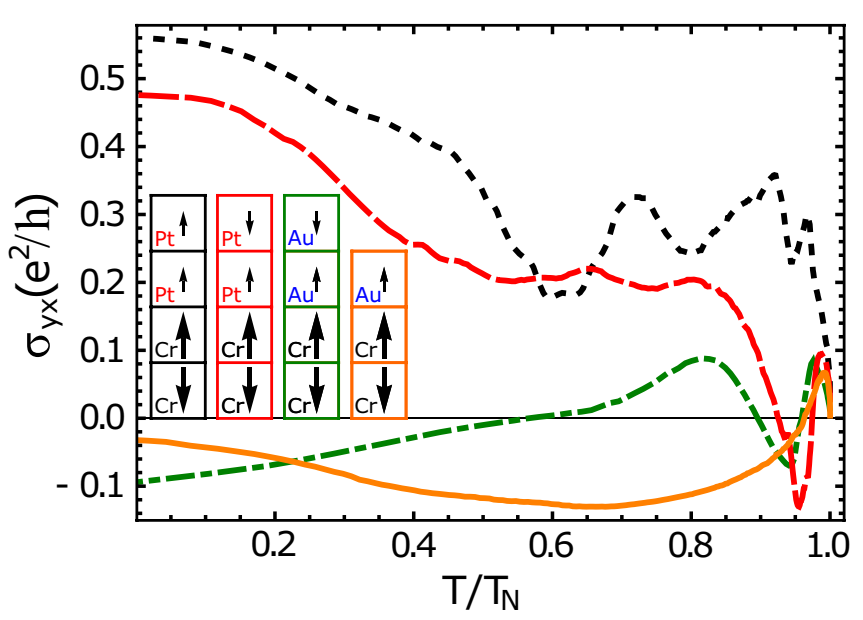

FIG. 4. Thermal dependence of the AHC obtained by computing the Berry curvature of the $d$ bands for the $\mathrm{Cr} / \mathrm{Pt}$ and $\mathrm{Cr} / \mathrm{Au}$ layered structures, assuming various magnetic profiles in the $\mathrm{Cr}, \mathrm{Pt}$, and $\mathrm{Au}$ interface layers. The proximized magnetization for $\mathrm{Pt}$ or $\mathrm{Au}$ has a smaller amplitude than that of $\mathrm{Cr}$, and it can have a ferromagnetic (dotted black) or antiferromagnetic (dashed red) profile, as sketched in the inset. The $\mathrm{Cr} / \mathrm{Au}$ interface corresponds to a physical configuration for the magnetization with a small (solid orange) or vanishing (dashed-dotted green) magnetization in the Au interface layers.

proximity should also be saturated because the sublattice antiferromagnetic magnetization has reached its maximal value.

Since uncompensated magnetization alone fails to account for these observations, an additional intrinsic contribution has to be considered. Concerning this term, one possible scenario would point to the presence of a noncollinear antiferromagnetic pattern developing at the $\mathrm{Pt} / \mathrm{Cr}$ interface. Such a possibility is, however, ruled out, as the theoretical investigation indicates a strong magnetic anisotropy and the Dzyaloshinskii-Moriya coupling is small at the $\mathrm{Cr} / \mathrm{Pt}$ interface [26]. For this reason we consider the possibility of having an intrinsic contribution due to a nontrivial Berry curvature in momentum space arising from the modification of the electronic structure due to the antiferromagnetic order and the magnetic proximity.

Hence we investigate a multiband low-energy tight-binding model which includes the bands at the Fermi level having $\mathrm{Pt}$ and $\mathrm{Cr}$ character, the $\mathrm{SOC}$ at the $\mathrm{Cr}$ site, and a magnetic interaction that takes into account the layer-dependent magnetization close to the interface both in the $\mathrm{Pt}$ and $\mathrm{Cr}$ regions. We then determine the intrinsic geometric contribution to the anomalous Hall conductance (AHC) by evaluating the Berry curvature of the Bloch bands. The AHC is proportional to the anomalous Hall resistance experimentally measured. The analysis is performed for different spatial profiles of the magnetization at the $\mathrm{Pt}$ side of the heterostructure with parallel or antiparallel magnetic moments in neighboring layers (see inset of Fig. 4). Moreover, in order to assess the role of an interface, a comparison is made by replacing Pt with Au. Since the magnetization in each layer is uniform, the temperaturedependence simulation of the AHC in the heterostructure can be performed by assuming a Stoner model with $M=$ $M_{0}\left[1-\left(\frac{T}{T_{N}}\right)^{2}\right]^{1 / 2}$, where $M_{0}$ is the zero-temperature mag- netization. Concerning the layer-dependent $M_{0}$ close to the interface, we take as reference the values obtained within the ab initio analysis.

Representative results for the AHC are shown in Fig. 4 where we compare three cases: (i) the $\mathrm{Cr} / \mathrm{Pt}$ interface with a sign modulation of the magnetization in the Pt layers (red curve), (ii) $\mathrm{Cr} / \mathrm{Pt}$ with a uniform penetration of the magnetization in the first layers close to the interface (black curve), and (iii) the $\mathrm{Cr} / \mathrm{Au}$ interface with weak ferromagnetic proximity (orange) or vanishing net magnetization (green) but higher electron density in the $\mathrm{Au}$ subsystem.

Although the Berry curvature is expected to be nonvanishing in the collinear antiferromagnet due to the breaking of the symmetry that combines time-reversal operation and half-unit-cell translation [5], here we report a careful analysis of the interface electronic states, indicating that they can also exhibit a topological character with a nontrivial associated Chern number (see Appendix G). Due to the presence of nontrivial sources of Berry curvature, we find that an overall positive amplitude of the AHC is generally obtained even with vanishing net magnetization in the Pt subsystem. On the other hand, for the case of the $\mathrm{Au} / \mathrm{Cr}$ interface the $\mathrm{AHC}$ tends to be negative below the magnetic transition temperature. Although a quantitative comparison between theory and experiments is beyond the scope of this analysis, the outcomes are robust and we argue that, due to its topological character (see Appendix G), the AHC does not exhibit a significant dependence on the detailed profile of the magnetic proximity. On the other hand, the occupation of the bands can affect the $\mathrm{AHC}$, as demonstrated by the tendency to exhibit opposite sign when comparing the $\mathrm{Pt} / \mathrm{Cr}$ with $\mathrm{Au} / \mathrm{Cr}$ bilayers.

Recently, it was shown that partially occupied $t_{2 g}$ bands, in the presence of SOC and ferromagnetism, can also generate AHE due to topological effects in momentum space [34]. Here, although we deal with antiferromagnets, similar electronic mechanisms are at work for the $\mathrm{Cr} / \mathrm{Pt}$ heterostructure with two effective sources for the AHE: the magnetic proximity of $\mathrm{Pt}$ and the $\mathrm{Cr}$ interface layers (see Appendix G). In bulk $\mathrm{Cr}$ the contributions to the AHC of different antiferromagnetic layers substantially cancel each other by symmetry. Although the AHC is not zero, gold is less effective in generating an AHE, because large onsite energy reduces the hybridization with interfacial Cr layers. Consistently, from our DFT calculations, gold does not show any intrinsic magnetic moment (see Appendix D). In this case we find that a sign change of the AHC can be obtained in a way that depends on the features of the magnetic proximity.

\section{CONCLUSIONS}

In conclusion, we have investigated and assessed the origin of the AHE in Cr-based heterostructure. The role of magnetic proximity has been carefully evaluated both experimentally and theoretically. DFT and electrical measurements agree on the existence of a spin-polarized $\mathrm{Pt}$ layer at the interface with $\mathrm{Cr}$ characterized by an out-ofplane magnetization. At the interface, $\mathrm{Cr}$ and $\mathrm{Pt}$ influence each other. In closer detail, the main effect of $\mathrm{Cr}$ on 


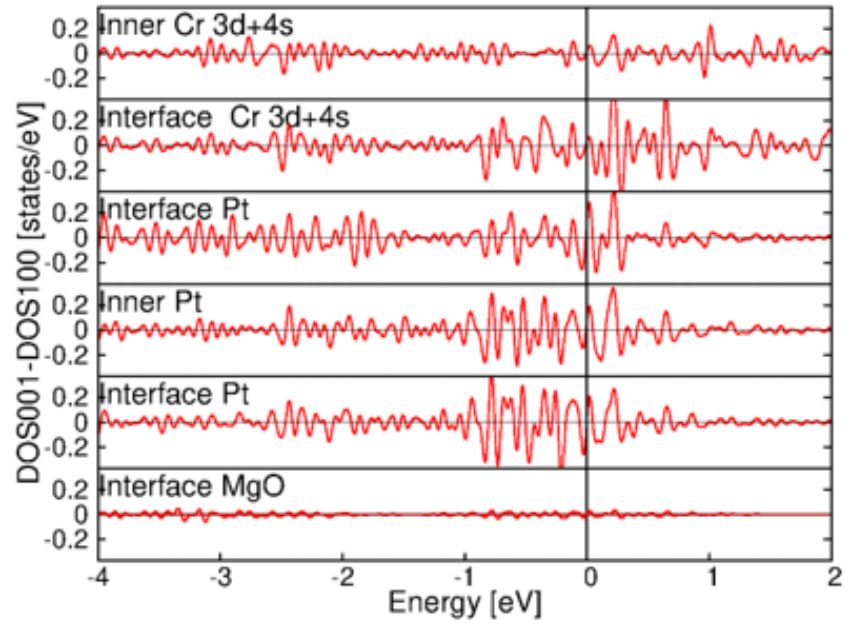

FIG. 5. Difference between the layer projected DOS for the system calculated with the out-of-plane (001) and in-plane (100) easy axes.

$\mathrm{Pt}$ is the induced magnetization profile. On the other hand, $\mathrm{Pt}$ induces a large MCA on the $\mathrm{Cr}$ side, favoring the OOP component of the magnetization. Exploiting the transport properties related to SDW ordering, we demonstrated a correspondence between AHE coming from the $\mathrm{Pt} / \mathrm{Cr}$ interface and AFM ordering in Cr. Finally, since the magnetic measurements exclude the presence of a net magnetization that can be associated to the proximity with the antiferromagnetic moments in the Cr system, we calculated the Berry curvature of the spin-polarized electronic states, showing that the intrinsic contribution to the AHE can justify the occurrence and temperature dependence of the AHE.

Whereas the results reported in this paper highlight the specific role of the interface with $\mathrm{Pt}$, we foresee similar physical scenarios with other nonmagnetic metals reported to have some proximity magnetization like $\mathrm{Pd}$ [35], Ir, W [11], or $\mathrm{V}$ [36], thus making $\mathrm{Cr}$ a paradigmatic test bed to study these interface effects. At the same time, we expect AHE coming from interfaces to be generally exploitable in a large variety of antiferromagnetic systems, either metallic or insulating, making Hall measurements a powerful probe of antiferromagnetism, both for fundamental studies and in view of applications.

\section{ACKNOWLEDGMENTS}

We thank R. Bertacco, X. Marti, D. Di Sante, M. Bragato, S. Achilli, and M. I. Trioni for fruitful discussion. This work was partially performed at Polifab, the micro and nanofabrication facility of Politecnico di Milano. The work is supported by Fondazione Cariplo via the project Magister (Project No. 2013-0726) and the Foundation for Polish Science through the IRA program cofinanced by the EU within SG OP. C.A. was supported by CNR-SPIN via the Seed Project CAMEO. W.B. acknowledges partial support by Narodowe Centrum Nauki (NCN, National Science Centre, Poland) Project No. 2016/23/B/ST3/00839. We acknowledge the CINECA Award under the ISCRA initiative IsC43 "C-MONAMI" and an IsC54 "CAMEO" grant, and for the availability of high-performance computing resources and support.

\section{APPENDIX A: THE DOS OF THE Cr/Pt FOR DIFFERENT SPIN ORIENTATION}

To study the properties of the surface, we add $20 \AA$ of vacuum to settle a setup for accurate calculations. We optimize the internal degrees of freedom by minimizing the total energy to be less than $10^{-6} \mathrm{eV}$ and the remaining forces to be less than $20 \mathrm{meV} / \AA$. A $16 \times 16 \times 1 k$-point MonkhorstPack grid is used for the relaxation and the calculation of the magnetocrystalline anisotropy. In addition, we use a $20 \times$ $20 \times 1 k$ point for the determination of the density of states (DOS) with a smearing of $0.05 \mathrm{eV}$ and an energy grid with a step of $0.1 \mathrm{meV}$. The SOC is included to calculate the magnetocrystalline anisotropy as a function of the lattice constant. We calculate the layer-projected DOS for the $\mathrm{MgO} / \mathrm{Pt} / \mathrm{Cr}$ heterostructure to make an interface of the $\mathrm{Pt}$ with the $\mathrm{Cr}$ and the $\mathrm{MgO}$. The plot of the difference between the DOS of the system with in-plane and out-of-plane easy axes is reported in Fig. 5.

The width of the oscillation is proportional to the effect of the SOC in the different layers. Large oscillations are present in any $\mathrm{Pt}$ layer. The interface $\mathrm{MgO}$ does not show any oscillations. The $\mathrm{Cr}$ layers show large oscillations for the interface
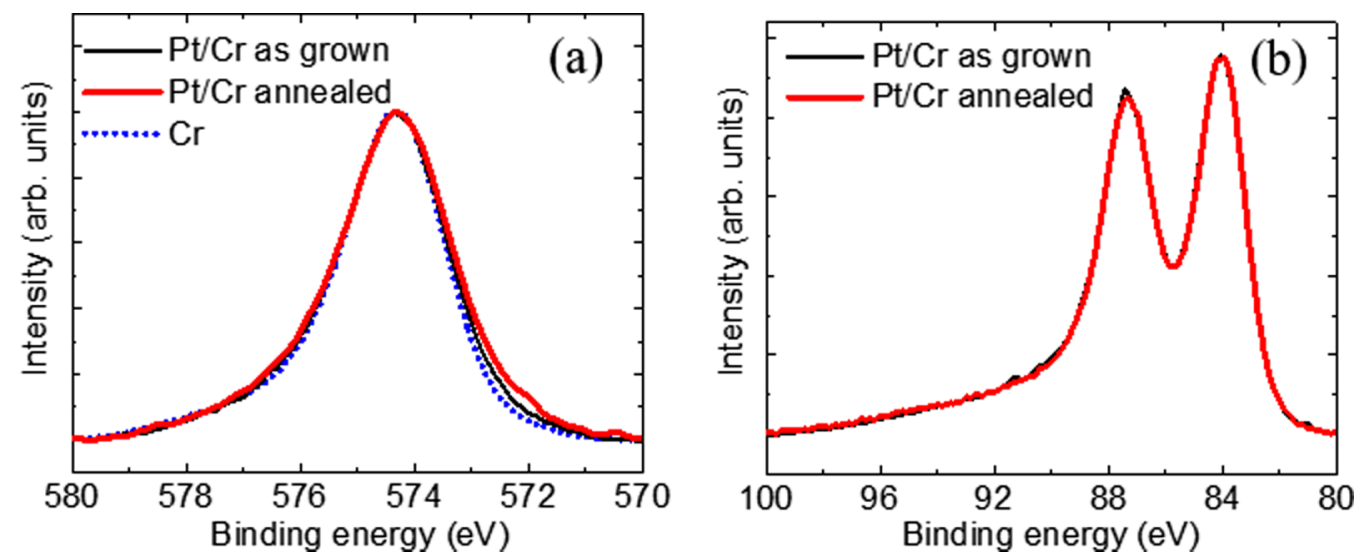

FIG. 6. XPS spectra of $\mathrm{Cr} 2 p_{3 / 2}$ (a) and Pt $4 f$ (b) core levels before and after Pt deposition and annealing. 

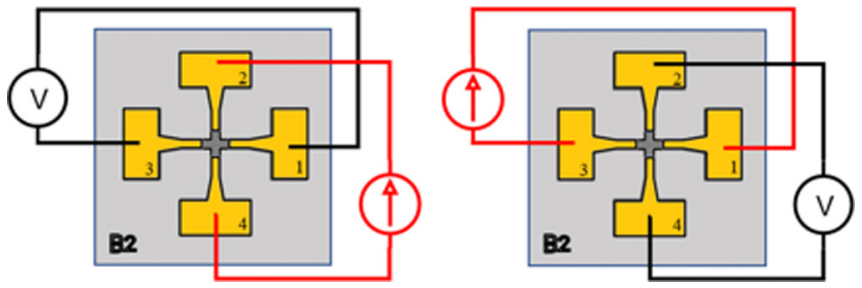

FIG. 7. Transverse resistivity measurement configuration in Hall crosses.

layer and smaller oscillations for the inner layers. Therefore the Pt induces a large SOC on the interface layer, and the contribution to the MCA mainly comes from this first layer of $\mathrm{Cr}$ interfaced with $\mathrm{Pt}$.

\section{APPENDIX B: GROWTH AND CHEMICAL CHARACTERIZATION OF Pt/Cr SAMPLES}

$\mathrm{Pt} / \mathrm{Cr}$ bilayers were grown on $\mathrm{MgO}(001)$ substrates in UHV pressure $\left(<10^{-9} \mathrm{mbar}\right)$ in a cluster tool allowing for in situ substrate cleaning, sample growth by molecular beam epitaxy (MBE), and characterization by x-ray photoelectron spectroscopy (XPS) and diffraction (XPD), as reported in detail elsewhere [37]. $\mathrm{Cr}$ and $\mathrm{Pt}$ were deposited by MBE at about $0.3 \mathrm{~nm} / \mathrm{min}$, as checked by a quartz microbalance. The substrate was kept at $373 \mathrm{~K}$ during $\mathrm{Cr}$ deposition, and then a post-annealing at $873 \mathrm{~K}$ was performed for improving film ordering and strain relaxation [21]. Pt was grown at room temperature (RT) and post-annealed at $473 \mathrm{~K}$. From x-ray diffraction data, the residual strain in $\mathrm{Cr}$ is less than $0.4 \%$ even in the thinnest film; thus the lattice parameter of $\mathrm{Cr}$ is largely comparable to the bulk case.

The samples were chemically characterized by XPS. Photoelectrons were excited by standard $\mathrm{Al} K \alpha$ x-ray source and collected by a hemispherical energy analyzer (HEA) Phoibos 150 (SPECS), yielding an acceptance angle of $\pm 2.5^{\circ}$, a field view of $\sim 1.4 \mathrm{~mm}^{2}$, and an energy resolution of $0.95 \mathrm{eV}$. Spectra were acquired at room temperature and normal incidence.

Figure 6 reports the $\mathrm{Cr} 2 p_{3 / 2}$ (left) and $\mathrm{Pt} 4 f$ (right) core levels before Pt deposition (blue dots), after Pt deposition (thin black line), and after Pt annealing (thick red line). The $\mathrm{Cr} 2 p_{3 / 2}$ core-level peak is quite identical for a $\mathrm{Cr}$ film (blue dots), the as-grown $\mathrm{Pt} / \mathrm{Cr}$ (thin black line), and $\mathrm{Pt} / \mathrm{Cr}$ after annealing at $473 \mathrm{~K}$ (thick red line), and the absence of chemical shifts suggests the chemical stability and absence of interdiffusion at the interface. A detailed analysis is reported in Ref. [21], in which the high crystallinity of the Pt and $\mathrm{Cr}$ layers is proved.

\section{APPENDIX C: ELECTRICAL MEASUREMENTS IN Pt/Cr}

Both longitudinal and transverse resistance measurements were performed as a function of temperature using a currentsource/nanovoltmeter pair (Keithley 6221/2182A) operating in Delta mode. Essentially, the Delta mode automatically triggers the current source to alternate the signal polarity and then triggers a nanovoltmeter reading at each polarity. This current reversal technique cancels out constant thermoelectric offsets, ensuring the results reflect the true value of the voltage. A relay switching matrix was used to acquire both transverse and

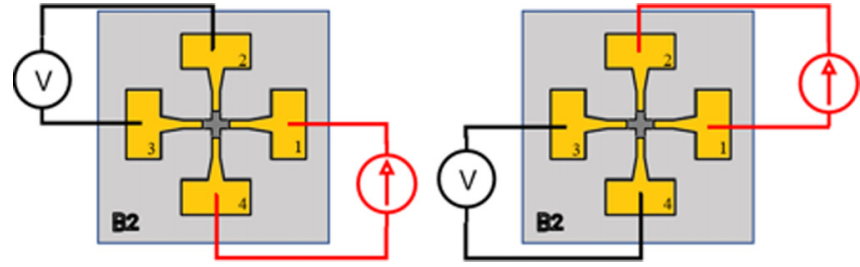

FIG. 8. Van der Pauw configuration for the measurement of resistivity in Hall crosses.

longitudinal measurements at the same time. The two contacts configurations required for geometrical offset subtraction [22] are pictured in Fig. 7. The van der Pauw measurements of resistivity [38] were performed using the two configurations shown in Fig. 8. In general, the intrinsic capability of this method of automatically subtracting offset terms of different origin (geometrical, thermal, etc.) makes it viable for fast and low-noise electrical characterization of phenomena where the signal to be measured is comparable to or lower than the offset itself.

Representative measurements of transverse resistance $\left(R_{x y}\right.$ in the main text) in $\mathrm{Pt}(3) / \mathrm{Cr}(75)$ are shown in Fig. 9. Here both geometrical and thermal offset compensation techniques are applied. Nevertheless, a temperature-dependent offset of nonmagnetic origin, whose amplitude is often comparable to the small magnetic signal coming from the $\mathrm{Pt} / \mathrm{Cr}$ interface, is observed. This uncompensated offset is different for each sample and presumably comes from minor nonuniformities in the sample. This spurious signal is fully reproducible over multiple subsequent measurements and can be subtracted as for the data reported in Figs. 2(c) and 3 of the main text.

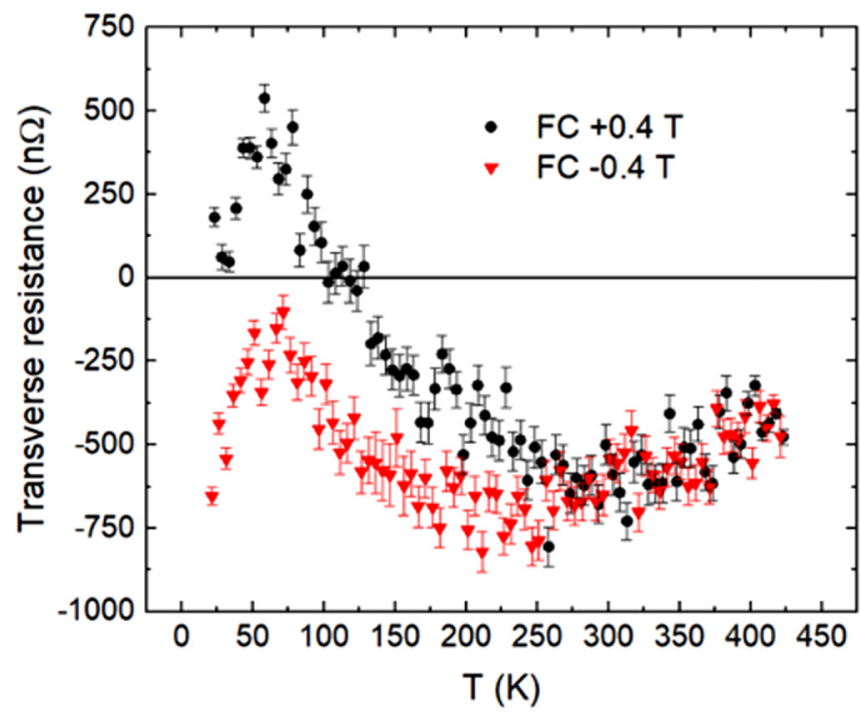

FIG. 9. Raw measurement of transverse resistance measured in $\mathrm{P}(3 \mathrm{~nm}) / \mathrm{Cr}(75 \mathrm{~nm})$ following field cooling at $+0.4 \mathrm{~T}$ (black points) and at $-0.4 \mathrm{~T}$ (red triangles). 

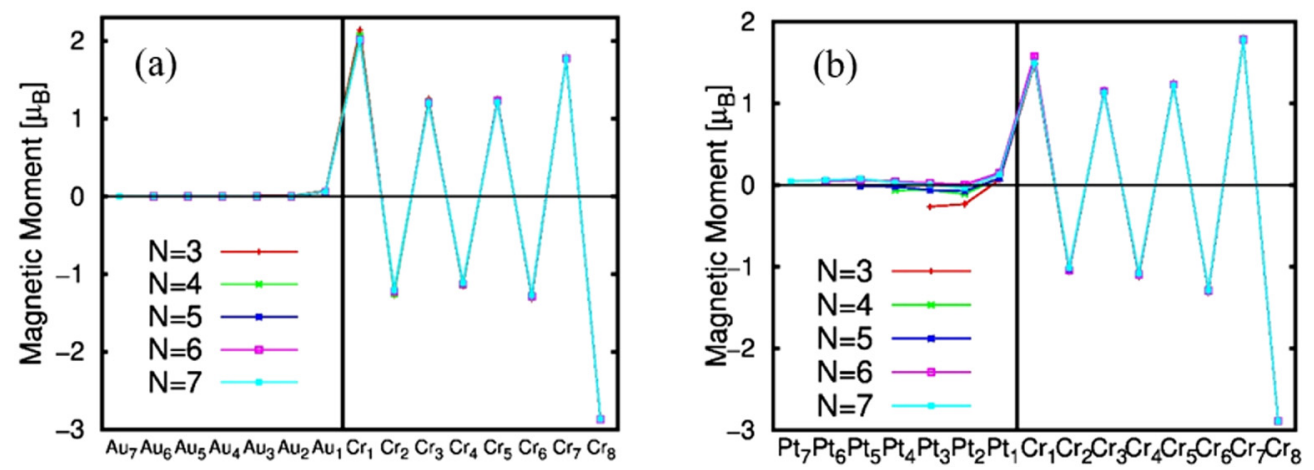

FIG. 10. Entire magnetic profile for the $\mathrm{Au} / \mathrm{Cr}(\mathrm{a})$ and $\mathrm{Pt} / \mathrm{Cr}(\mathrm{b})$ heterostructure. $\mathrm{The} \mathrm{Cr}_{1}\left(\mathrm{Pt}_{1}, \mathrm{Au}_{1}\right)$ is the $\mathrm{Cr}(\mathrm{Pt}$, $\mathrm{Au})$ interface layer, and the $\mathrm{Cr}_{i}\left(\mathrm{Pt}_{i}, \mathrm{Au}_{i}\right)$, with $i>1$, is the $i$ th layer from the interface.

\section{APPENDIX D: THE MAGNETIC PROFILE FOR THE Pt/Cr AND Au/Cr INTERFACES}

The entire profile of the $\mathrm{Pt}(\mathrm{N}) / \mathrm{Cr}$ and $\mathrm{Au}(\mathrm{N}) / \mathrm{Cr}$ magnetizations is reported in Fig. 10. The $\mathrm{Cr}$ magnetic moment is almost independent from the number of the $5 d$ layers. At the surface, the magnetic moment is close to $2 \mu_{B}$ and the magnetization of the inner layers oscillates. The main difference in the $\mathrm{Cr}$ layers between the two $5 d$ systems is the different magnetization of the interface that is larger for the Au interface.

A magnification of the magnetic profile of the $\mathrm{Au} / \mathrm{Cr}$ heterostructure is shown in panel (a) of Fig. 11. The interface gold layer presents a small magnetization of $0.06 \mu_{B}$ independently of the Au thickness, while no magnetization is present for the other layers. Since this magnetization goes rapidly to zero far from the $\mathrm{Cr}$ layers, we propose that magnetization of the interface $\mathrm{Au}$ is not related to the intrinsic magnetic moment of gold but is simply due to the tails of the magnetization of the $\mathrm{Cr}$ atoms. The AHE measured for the $\mathrm{Au} / \mathrm{Cr}$ system can be entirely attributed to the $\mathrm{Cr}$ interface. This also explains the smaller value of the AHE that arises from one single Au layer, while in the case of the $\mathrm{Pt} / \mathrm{Cr}$ system many Pt layers contribute to the AHE. The different sign of the AHE between $\mathrm{Pt} / \mathrm{Cr}$ and $\mathrm{Au} / \mathrm{Cr}$ also suggests that the main contribution to the two cases is different.

A magnification of the magnetic profile of the $\mathrm{Pt} / \mathrm{Cr}$ heterostructure is shown in panel (b) of Fig. 11. The total magnetic moment of the $\mathrm{Pt}$ region is $-0.429,-0.147,-0.084$, 0.336 , and $0.315 \mu_{\mathrm{B}}$, respectively, for $N=3,4,5,6,7$. The total magnetic moment of the Au region, instead, is basically always equal to the induced magnetization of $0.06 \mu_{B}$.

\section{APPENDIX E: SQUID CHARACTERIZATION OF Pt/Cr SAMPLES}

To gain insight into the magnetic proximity effect, fielddependent magnetization measurements were performed on $\mathrm{Pt}(2) / \mathrm{Cr}(50)$ samples by using a superconductive quantum interference device-vibrating sample magnetometer (SQUID-VSM, LOT- Quantum Design) with high sensitivity $\left(\leqslant 10^{-11} \mathrm{~A} \mathrm{~m}^{2}\right.$ ) and equipped with both large field (up to $7 \mathrm{~T}$ ) and a wide temperature range $(2.5-1000 \mathrm{~K})$.

Figure 12 shows the magnetization versus $H$ measured at different temperatures (in the 5-320 K range) after field cooling the samples from $320 \mathrm{~K}$ under a perpendicular magnetic field of $+0.4 \mathrm{~T}$. After subtracting a linear diamagnetic contribution, only a ferromagnetic contribution remains, rather constant in the investigated temperature range, below and above the Néel temperature of the antiferromagnet. Any signal related to the AFM phase transition should have disappeared above the Néel temperature of the film $(290 \pm 5 \mathrm{~K})$. Therefore we ascribe such a contribution to substrate impurities. Note
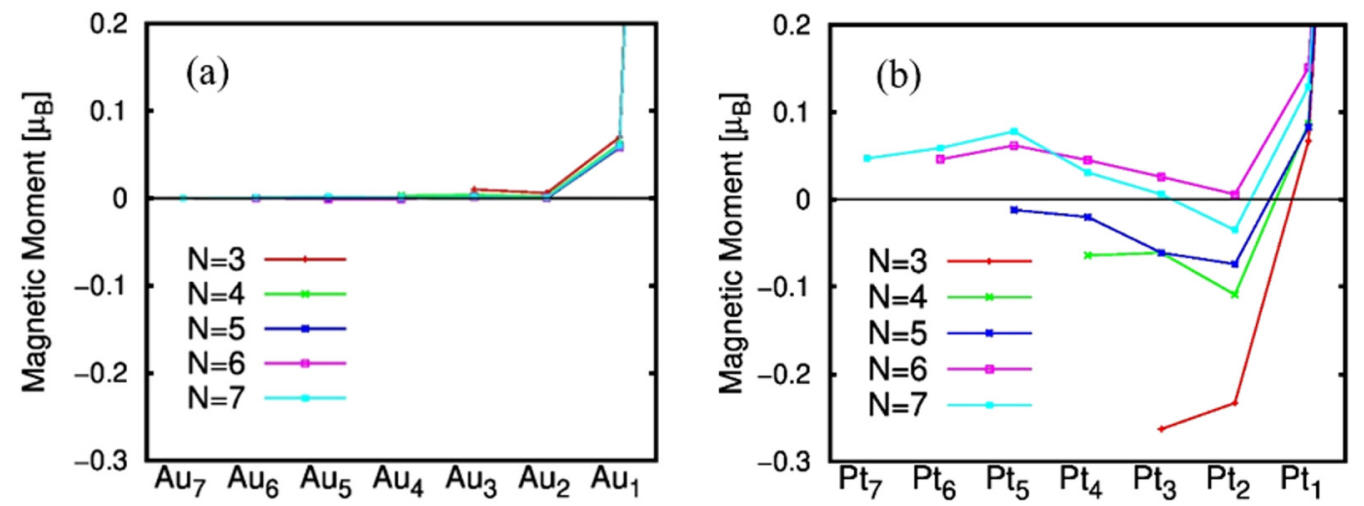

FIG. 11. Magnetic profile of the (a) $\mathrm{Au}(\mathrm{N}) / \mathrm{Cr}$ interface and (b) $\mathrm{Pt}(\mathrm{N}) / \mathrm{Cr}$ interface. The $\mathrm{Au}_{1}\left(\mathrm{Pt}_{1}\right)$ is the $\mathrm{Au}(\mathrm{Pt})$ interface layer, and the $\mathrm{Au} i$ $\left(\mathrm{Pt}_{i}\right)$ is the $i$ th layer from the interface. 


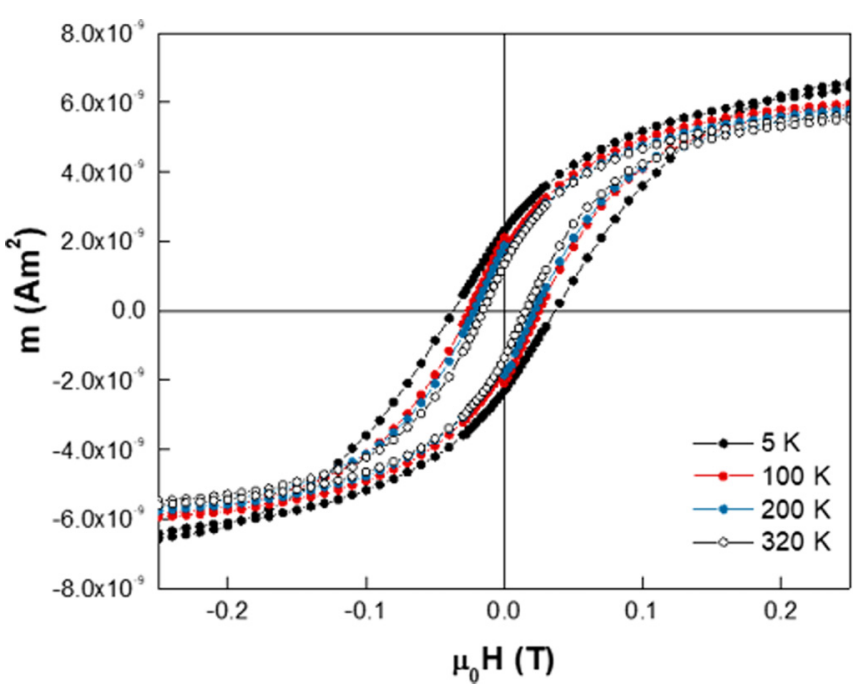

FIG. 12. Magnetic moment versus field loops at different temperatures measured in remanence after field cooling the sample with magnetic field of $0.4 \mathrm{~T}$ perpendicular to the surface.

that this magnetic signal does not have a counterpart in the detected electrical signals.

In order to check the presence of proximity effects at the $\mathrm{Pt} / \mathrm{Cr}$ interface by magnetometry, we repeated the measurements after cooling the samples under a perpendicular magnetic field of opposite sign $(-0.4 \mathrm{~T})$. No difference emerged between the two field coolings. The result is not unexpected, taking into account the calculations reported in Fig. 11 of Appendix D. Indeed, the expected proximityinduced magnetic moment is around $0.3 \mu \mathrm{B}$ /atom for the second Pt layer, antiferromagnetically coupled with $\mathrm{Cr}$. For the first layer it is $\sim 0.05 \mu \mathrm{B} /$ atom, with ferromagnetic coupling. If only the latter, and not the whole film, would be blocked by the proximity effect, the contribution would be correspondingly reduced and so the measured moment. Moreover, the overall induced magnetic moment integrated on the sample area can be reduced by the fact that the AFM is probably broken into different domains, since the field-cooling process is not able to completely reorient the AFM [39]. For these reasons the magnetic signal versus field cooling is dominated by spurious contributions and not by the magnetic induced moments. The result shows that the electrical readout is very effective to detect the magnetic moment induced by proximity at the interface.

\section{APPENDIX F: COMPARISON OF TRANSPORT IN Pt/Cr AND Au/Cr}

In Fig. 3 of the main text we compared $\mathrm{Pt}(3) / \mathrm{Au}(3)$ and $\operatorname{Pt}(3)$ capping layers on top of the same $\operatorname{Cr}(50)$ thin film. To foster the conclusion that the different transverse resistance signal at remanence is related only to the different interface, we present here the transport properties of the whole heterostructure (largely dominated by the chromium layer), indicating in both cases a solid antiferromagnetic behavior below $290 \mathrm{~K}$.

Figure 13 reports the longitudinal resistivity (full dots) and its first derivative for Au-capped (left) and Pt-capped (right) chromium devices after a field cooling at $+0.4 \mathrm{~T}$ (upward field) and $-0.4 \mathrm{~T}$ (downward field). In both cases the local maximum in the first derivative (empty dots) observed at $290 \mathrm{~K}$ can be used as a reference for the antiferromagnetic transition of $\mathrm{Cr}$. The same signature of the gap opening arising from the spin-density wave in $\mathrm{Cr}$ can be observed as well, independently of the nature of the interface, in the carrier density extrapolated from Hall measurements in applied magnetic field $(B= \pm 0.4 \mathrm{~T})$ shown in Fig. 14.

\section{APPENDIX G: BERRY CURVATURE OF THE INTERFACIAL ELECTRONIC STATES AND TOPOLOGICAL CHARACTER}

The intrinsic contribution to the anomalous Hall conductivity is dependent on the band structure of the crystal through the Berry curvature of the Bloch bands labeled by the index $n$ as

$$
\sigma_{i j}^{\mathrm{AH}-\mathrm{int}}=-\varepsilon_{i j l} \frac{e^{2}}{\hbar} \sum_{n} \int \frac{1}{(2 \pi)^{2}} d \mathbf{k} f\left[E_{n}(\mathbf{k})\right] \Omega_{n}(\mathbf{k}),
$$

where $\varepsilon_{i j l}$ is the Levi-Civita tensor, $f\left[E_{n}(\mathbf{k})\right]$ is the Fermi distribution function for the band $E_{n}(\mathbf{k})$, and for a given eigenstate $\left|n_{\mathbf{k}}\right\rangle$, one has that $a_{n}(\mathbf{k})$ is the Berry connection
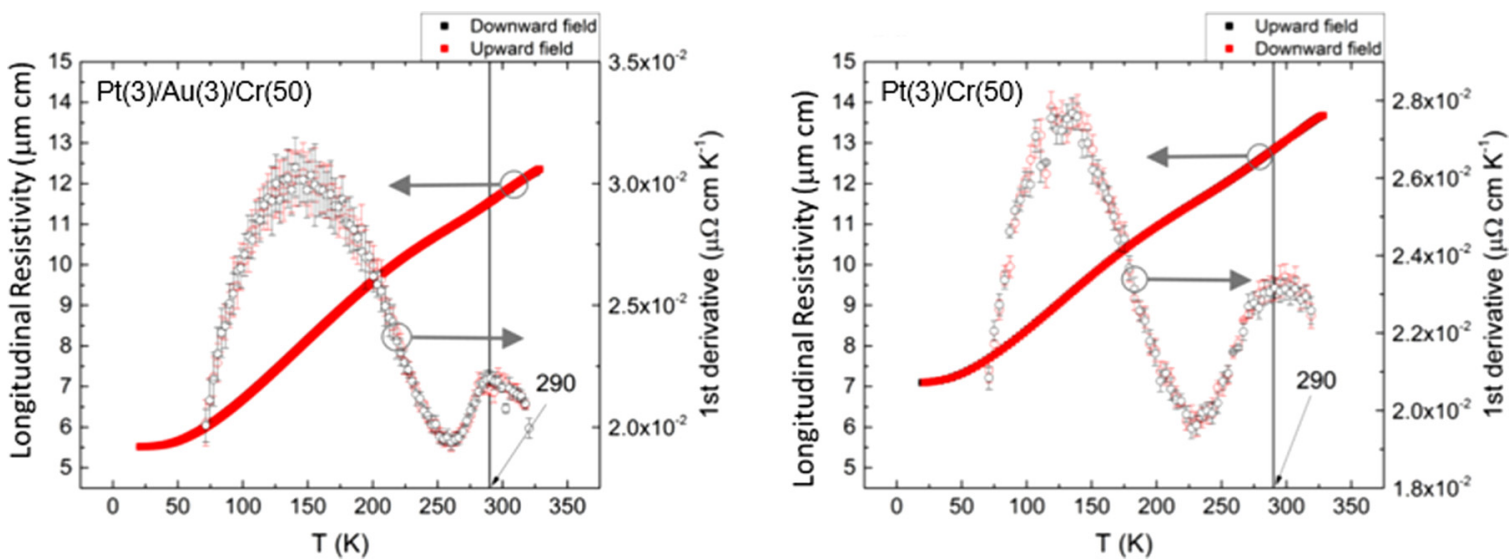

FIG. 13. Longitudinal resistivity and its first derivative in $\mathrm{Pt}(3) / \mathrm{Au}(3) / \mathrm{Cr}(50)$ (left) and $\mathrm{Pt}(3) / \mathrm{Cr}(50)$ (right). 

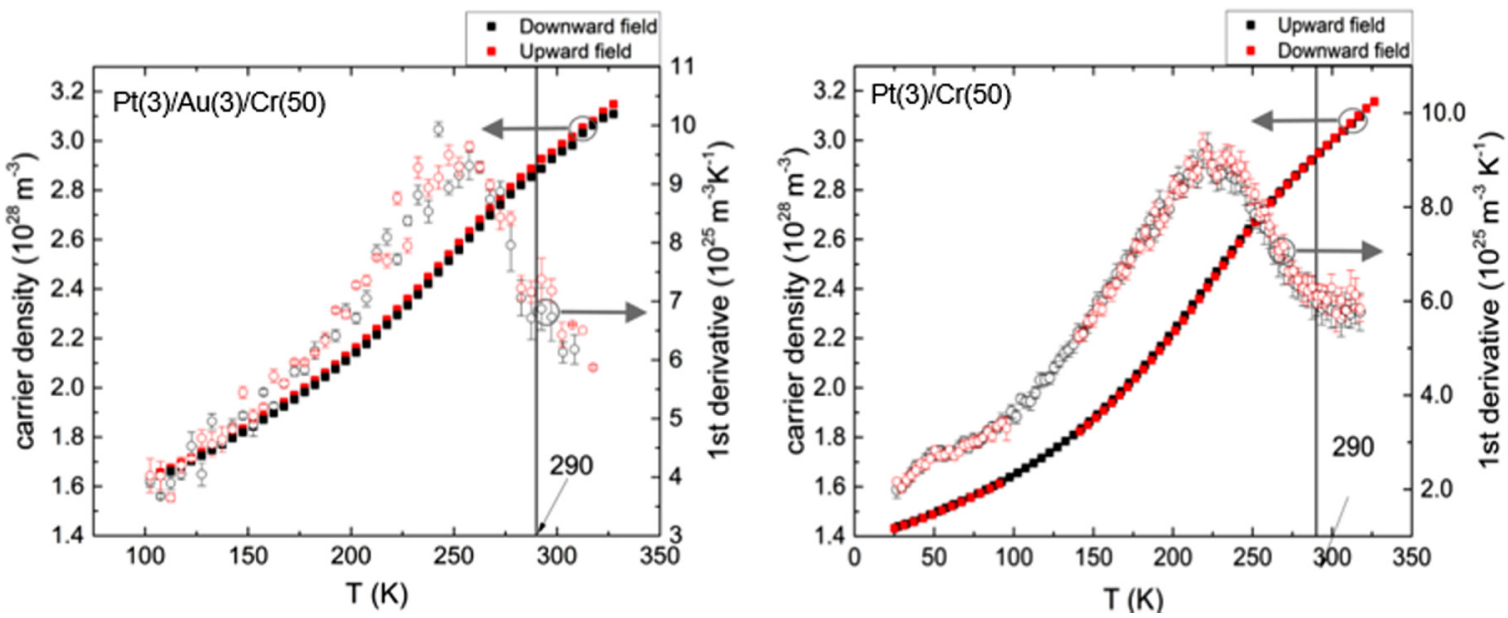

FIG. 14. Carrier density and its first derivative in $\mathrm{Pt}(3) / \mathrm{Au}(3) / \mathrm{Cr}(50)$ (left) and $\mathrm{Pt}(3) / \mathrm{Cr}(50)$ (right).

$a_{n}(\mathbf{k})=i\left\langle n_{\mathbf{k}}\left|\nabla_{\mathbf{k}}\right| n_{\mathbf{k}}\right\rangle$, while $\Omega_{n}(\mathbf{k})$ is the Berry-phase curvature, $\Omega_{n}(\mathbf{k})=\nabla \times a_{n}(\mathbf{k})$. The Berry curvature is then evaluated from the Bloch eigenstate for each band by following the scheme of computation reported in Ref. [40], introducing a sufficiently fine mesh in the Brillouin zone according to the required accuracy.

In the main text we have determined the anomalous Hall conductivity by evaluating the Berry curvature contribution of each occupied band (below the Fermi level), taking into account an effective tight-binding model with elec- tronic parameters that follow the inputs from the $a b$ initio calculation.

Here we aim to show that the electronic structure of the $d$ bands at the $\mathrm{Cr} / \mathrm{Au}$ or $\mathrm{Cr} / \mathrm{Pt}$ interface can exhibit an intrinsic topological character corresponding to the occurrence of nontrivial Chern number. For this purpose we take a representative and minimal configuration based on a $\mathrm{Cr} / \mathrm{Au}$ bilayer assuming different amplitudes for the spin polarization of the Cr electronic states and for the induced magnetization in the $\mathrm{Au}$ layer. For the system upon examination there are 12 bands,

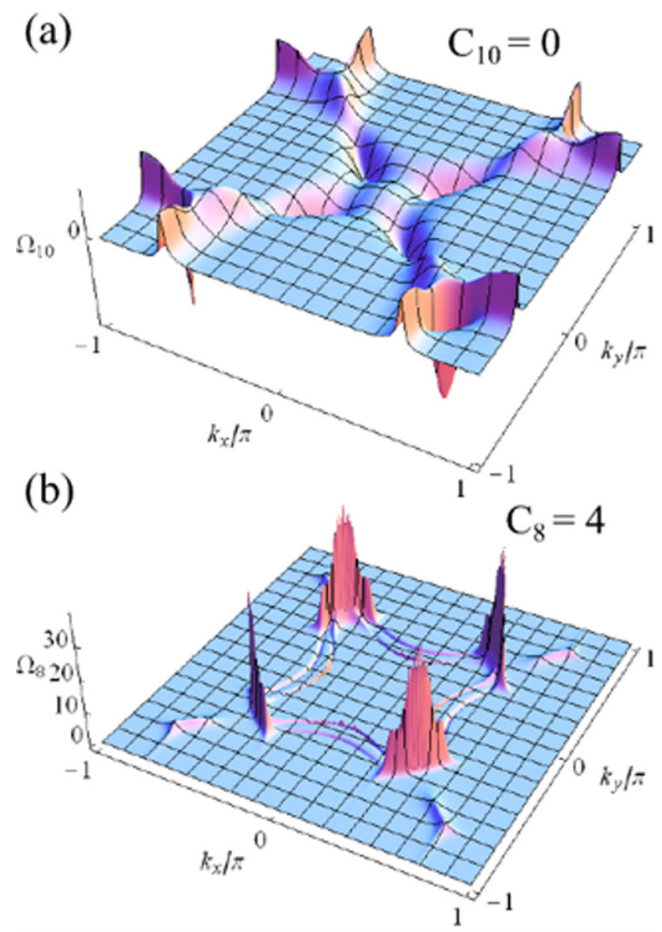

(c)

$$
\mathrm{C}_{10}=1
$$

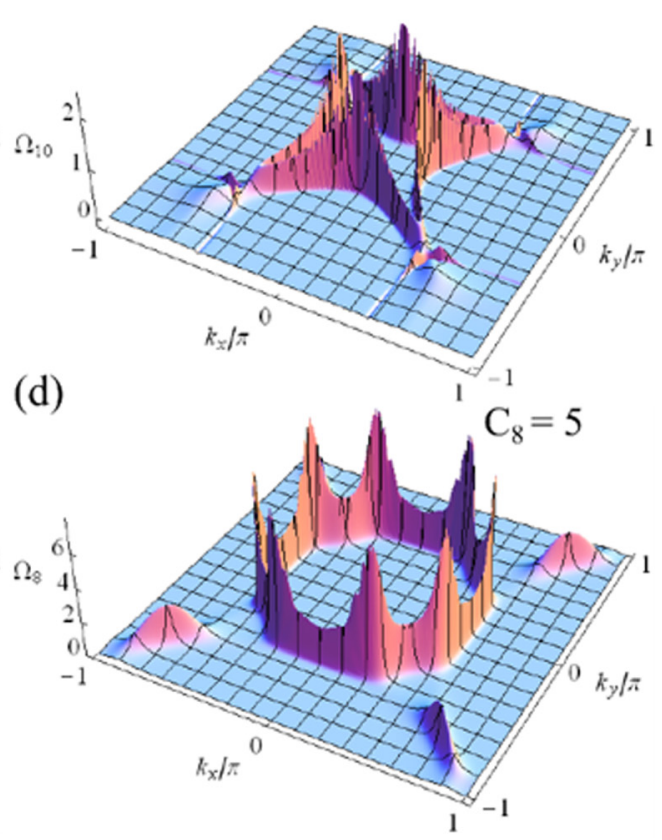

FIG. 15. Momentum-resolved profile of the Berry curvature $\Omega_{n}(\mathbf{k})$ for two representative bands $(n=8$ and 10) of the Cr-Au bilayer system. The magnetizations $M$ at the $\mathrm{Cr}$ and Au site are along the $z$ direction and have opposite sign. (a), (b) Small proximized magnetic moment $\left(M_{\mathrm{Au}}=0.05 t / U\right)$ with an intermediate amplitude of the spin polarization at the $\mathrm{Cr}$ site $\left(M_{\mathrm{Cr}}=-0.5 t / U\right)$. Here, $U$ is the local Coulomb interaction. (c), (d) Larger proximity in the Au layer $\left(M_{\mathrm{Au}}=0.1 t / U\right)$ with stronger magnetization at the $\mathrm{Cr}$ site, $M_{\mathrm{Cr}}=-1 t / U$. The corresponding Chern numbers are indicated as $C_{n}$. The electronic parameters are in-plane hoppings, $t_{1}=-t$ (nearest neighbor), $t_{2}=-0.16 t$ (next-nearest neighbor), interplane hoppings, $t_{1}=-0.7 t, t_{2}=-0.11 t$, spin-orbit coupling $\lambda_{\mathrm{Cr}}=-0.05 t, \lambda_{\mathrm{Au}}=-0.5 t$. 
and one can determine both the Berry curvature for the corresponding electronic configurations and the integrated value $C_{n}=\int \frac{1}{(2 \pi)^{2}} d \mathbf{k} \Omega_{n}(\mathbf{k})$. In Fig. 15 we report few characteristic cases of momentum-resolved Berry curvature for a set of magnetic configurations with the magnetic moment at the $\mathrm{Cr}$ atom being larger and antiparallel with respect to that of the Au site. As one can notice, depending on the strength of the magnetization at the $\mathrm{Cr}$ and $\mathrm{Au}$ site, one can drive a changeover of the topological number in the system. This is demonstrated for two representative bands where the corresponding Chern number is changed from 0 to 1 or from 4 to 5 . Such behavior is nontrivial and indicates the intrinsic topological character of the electronic states at the $\mathrm{Cr}$ interface. Furthermore, although in the metallic configurations there are only portions of the Berry curvature contributing to the anomalous Hall effect, we expect that cancellation effects due to interband interference are not likely to occur and can only manifest with electronic fine tuning.
[1] N. Nagaosa, J. Sinova, S. Onoda, A. H. MacDonald, and N. P. Ong, Anomalous Hall effect, Rev. Mod. Phys. 82, 1539 (2010).

[2] S. Nakatsuji, N. Kiyohara, and T. Higo, Large anomalous Hall effect in a non-collinear antiferromagnet at room temperature, Nature (London) 527, 212 (2015).

[3] A. K. Nayak, J. E. Fischer, Y. Sun, B. Yan, J. Karel, A. C. Komarek, C. Shekhar, N. Kumar, W. Schnelle, J. Ku bler, C. Felser, and S. S. P. Parkin, Large anomalous Hall effect driven by a nonvanishing berry curvature in the noncolinear antiferromagnet $\mathrm{Mn}_{3} \mathrm{Ge}$, Sci. Adv. 2, e1501870 (2016).

[4] T. Jungwirth, X. Marti, P. Wadley, and J. Wunderlich, Antiferromagnetic spintronics, Nat. Nanotechnol. 11, 231 (2016).

[5] L. Šmejkal, R. González-Hernández, T. Jungwirth, and J. Sinova, Crystal Hall effect in collinear antiferromagnets, arXiv:1901.00445.

[6] S. Y. Huang, X. Fan, D. Qu, Y. P. Chen, W. G. Wang, J. Wu, T. Y. Chen, J. Q. Xiao, and C. L. Chien, Transport Magnetic Proximity Effects in Platinum, Phys. Rev. Lett. 109, 107204 (2012).

[7] E. Fawcett, Spin-density-wave antiferromagnetism in chromium, Rev. Mod. Phys. 60, 209 (1988).

[8] G. Schütz, S. Stähler, M. Knülle, P. Fischer, S. Parkin, and $\mathrm{H}$. Ebert, Distribution of magnetic moments in $\mathrm{Co} / \mathrm{Pt}$ and $\mathrm{Co} / \mathrm{Pt} / \mathrm{Ir} / \mathrm{Pt}$ multilayers detected by magnetic $\mathrm{x}$-ray absorption, J. Appl. Phys. 73, 6430 (1993).

[9] W. Antel, M. M. Schwickert, T. Lin, W. L. O’Brien, and G. R. Harp, Induced ferromagnetism and anisotropy of Pt layers in Fe/Pt(001) multilayers, Phys. Rev. B 60, 12933 (1999).

[10] F. Wilhelm, P. Poulopoulos, G. Ceballos, H. Wende, K. Baberschke, P. Srivastava, D. Benea, H. Ebert, M. Angelakeris, N. K. Flevaris, D. Niarchos, A. Rogalev, and N. B. Brookes, Layer-Resolved Magnetic Moments in Ni/Pt Multilayers, Phys. Rev. Lett. 85, 413 (2000).

[11] F. Wilhelm, P. Poulopoulos, H. Wende, A. Scherz, K. Baberschke, M. Angelakeris, N. K. Flevaris, and A. Rogalev, Systematics of the Induced Magnetic Moments in $5 d$ Layers and the Violation of the Third Hund's Rule, Phys. Rev. Lett. 87, 207202 (2001).

[12] Y. M. Lu, Y. Choi, C. M. Ortega, X. M. Cheng, J. W. Cai, S. Y. Huang, L. Sun, and C. L. Chien, Pt Magnetic Polarization on $\mathrm{Y}_{3} \mathrm{Fe}_{5} \mathrm{O}_{12}$ and Magnetotransport Characteristics, Phys. Rev. Lett. 110, 147207 (2013).

[13] S. Geprägs, S. Meyer, S. Altmannshofer, M. Opel, F. Wilhelm, A. Rogalev, R. Gross, and S. T. B. Goennenwein, Investigation of induced $\mathrm{Pt}$ magnetic polarization in $\mathrm{Pt} / \mathrm{Y}_{3} \mathrm{Fe}_{5} \mathrm{O}_{12}$ bilayers, Appl. Phys. Lett. 101,262407 (2012).

[14] T. Kosub, M. Kopte, F. Radu, O. G. Schmidt, and D. Makarov, All-Electric Access to the Magnetic-Field-Invariant Magnetization of Antiferromagnets, Phys. Rev. Lett. 115, 097201 (2015).

[15] T. Kosub, M. Kopte, R. Hühne, P. Appel, B. Shields, P. Maletinsky, R. Hübner, M. O. Liedke, J. Fassbender, O. G. Schmidt, and D. Makarov, Purely antiferromagnetic magnetoelectric random access memory, Nat. Commun. 8, 13985 (2017).

[16] J. Mattson, B. Brumitt, M. B. Brodsky, and J. B. Ketterson, Magnetotransport studies of epitaxial $\mathrm{Cr}$ thin films, J. Appl. Phys. 67, 4889 (1990).

[17] R. K. Kummamuru and Y. Soh, Electrical effects of spin density wave quantization and magnetic domain walls in chromium, Nature (London) 452,859 (2008).

[18] G. Kresse and D. Joubert, From ultrasoft pseudopotentials to the projector augmented-wave method, Phys. Rev. B 59, 1758 (1999).

[19] J. P. Perdew and A. Zunger, Self-interaction correction to density-functional approximations for many-electron systems, Phys. Rev. B 23, 5048 (1981).

[20] P. E. Blöchl, Projector augmented-wave method, Phys. Rev. B 50, 17953 (1994).

[21] C. Rinaldi, M. Asa, D. Chrastina, J. L. Hart, M. L. Taheri, I. Pallecchi, D. Marré, and M. Cantoni, Study and optimization of epitaxial films of $\mathrm{Cr}$ and $\mathrm{Pt} / \mathrm{Cr}$ bilayers on $\mathrm{MgO}$, J. Phys. D. Appl. Phys. 53,105303 (2020).

[22] P. Daniil and E. Cohen, Low field Hall effect magnetometry, J. Appl. Phys. 53, 8257 (1982).

[23] A. Daire, W. Goeke, and M. A. Tupta, White Paper: New Instruments Can Lock out Lock-Ins, Keithley Instruments, Inc (2005).

[24] R. Hafner, D. Spišák, R. Lorenz, and J. Hafner, Magnetic ground state of $\mathrm{Cr}$ in density-functional theory, Phys. Rev. B 65, 184432 (2002).

[25] H. Yang, A. Thiaville, S. Rohart, A. Fert, and M. Chshiev, Anatomy of Dzyaloshinskii-Moriya Interaction at $\mathrm{Co} / \mathrm{Pt}$ Interfaces, Phys. Rev. Lett. 115, 267210 (2015).

[26] A. Belabbes, G. Bihlmayer, F. Bechstedt, S. Blügel, and A. Manchon, Hund's Rule-Driven Dzyaloshinskii-Moriya Interaction at $3 d-5 d$ Interfaces, Phys. Rev. Lett. 117, 247202 (2016). 
[27] S. Karoui, H. Amara, B. Legrand, and F. Ducastelle, Magnetism: The driving force of order in CoPt, a first-principles study, J. Phys.: Condens. Matter 25, 056005 (2013).

[28] S. S. P. Parkin, Systematic Variation of the Strength and Oscillation Period of Indirect Magnetic Exchange Coupling through the $3 d, 4 d$, and $5 d$ Transition Metals, Phys. Rev. Lett. 67, 3598 (1991).

[29] C. Andersson, B. Sanyal, O. Eriksson, L. Nordström, O. Karis, D. Arvanitis, T. Konishi, E. Holub-Krappe, and J. H. Dunn, Influence of Ligand States on the Relationship Between Orbital Moment and Magnetocrystalline Anisotropy, Phys. Rev. Lett. 99, 177207 (2007).

[30] Y.-T. Chen, S. Takahashi, H. Nakayama, M. Althammer, S. T. B. Goennenwein, E. Saitoh, and G. E. W. Bauer, Theory of spin Hall magnetoresistance, Phys. Rev. B 87, 144411 (2013).

[31] M. Isasa, E. Villamor, L. E. Hueso, M. Gradhand, and F. Casanova, Temperature dependence of spin diffusion length and spin Hall angle in Au and Pt, Phys. Rev. B 91, 024402 (2015).

[32] Y. Tian, L. Ye, and X. Jin, Proper Scaling of the Anomalous Hall Effect, Phys. Rev. Lett. 103, 087206 (2009).

[33] L. Ye, Y. Tian, X. Jin, and D. Xiao, Temperature dependence of the intrinsic anomalous Hall effect in nickel, Phys. Rev. B 85, 220403 (2012).
[34] D. J. Groenendijk, C. Autieri, T. C. van Thiel, W. Brzezicki, N. Gauquelin, P. Barone, K. H. W. van den Bos, S. van Aert, J. Verbeeck, A. Filippetti, S. Picozzi, M. Cuoco, and A. D. Caviglia, Berry phase engineering at oxide interfaces, Phys. Rev. Res. 2, 023404 (2020).

[35] D. O. Kim, K. M. Song, Y. Choi, B. C. Min, J. S. Kim, J. W. Choi, and D. R. Lee, Asymmetric magnetic proximity effect in a Pd/Co/Pd trilayer system, Sci. Rep. 6, 25391 (2016).

[36] T. Khajil and J. Khalifeh, Spin polarization of vanadium overlayers on a semi-infinite tantalum substrate, Surf. Sci. 389, 310 (1997).

[37] R. Bertacco, M. Cantoni, M. Riva, A. Tagliaferri, and F. Ciccacci, Epitaxial growth and characterization of layered magnetic nanostructures, Appl. Surf. Sci. 252, 1754 (2005).

[38] L. J. Van der Pauw, A method of measuring the resistivity and Hall coefficient on lamellae of arbitrary shape, Philips Res. Reports 13, 1 (1958).

[39] K. O'Grady, L. E. Fernandez-Outon, and G. Vallejo-Fernandez, A new paradigm for exchange bias in polycrystalline thin films, J. Mag. Mag. Mater. 322, 883 (2010).

[40] T. Fukui, Y. Hatsugai, and H. Suzuki, Chern numbers in discretized Brillouin zone: Efficient method of computing (spin) Hall conductances, J. Phys. Soc. Japan 74, 1674 (2005). 